Review

\title{
Risks of Using Sterilization by Gamma Radiation: The Other Side of the Coin
}

\author{
C. Randall Harrell ${ }^{1}$, Valentin Djonov ${ }^{2}$, Crissy Fellabaum ${ }^{1}$, and Vladislav Volarevic ${ }^{3} \llbracket$ \\ 1. Regenerative Processing Plant, LLC, Palm Harbor, Florida, United States of America. \\ 2. Institute of Anatomy, University of Bern, Bern, Switzerland. \\ 3. Department of Microbiology and Immunology, Center for Molecular Medicine and Stem Cell Research, Faculty of Medical Sciences, University of \\ Kragujevac, Kragujevac, Serbia. \\ $\triangle$ Corresponding author: Prof. dr Vladislav Volarevic; e mail: drvolarevic@yahoo.com; vladislav.volarevic@ana.unibe.ch; Faculty of Medical Sciences, \\ University of Kragujevac, Kragujevac, Serbia; 69 Svetozara Markovica Street, 34000 Kragujevac, Serbia \\ (C) Ivyspring International Publisher. This is an open access article distributed under the terms of the Creative Commons Attribution (CC BY-NC) license \\ (https://creativecommons.org/licenses/by-nc/4.0/). See http://ivyspring.com/terms for full terms and conditions
}

Received: 2017.09.01; Accepted: 2017.12.21; Published: 2018.01.18

\begin{abstract}
The standard sterilization method for most medical devices over the past 40 years involves gamma irradiation. During sterilization, gamma rays efficiently eliminate microorganisms from the medical devices and tissue allografts, but also significantly change molecular structure of irradiated products, particularly fragile biologics such as cytokines, chemokines and growth factors. Accordingly, gamma radiation significantly alters biomechanical properties of bone, tendon, tracheal, skin, amnion tissue grafts and micronized amniotic membrane injectable products. Similarly, when polymer medical devices are sterilized by gamma radiation, their physico-chemical characteristics undergo modification significantly affecting their clinical use.

Several animal studies demonstrated that consummation of irradiated food provoked genome instability raising serious concerns regarding oncogenic potential of irradiated consumables. These findings strongly suggest that new, long-term, prospective clinical studies should be conducted in near future to investigate whether irradiated food is safe for human consumption. In this review, we summarized current knowledge regarding molecular mechanisms responsible for deleterious effects of gamma radiation with focusing on its significance for food safety and biomechanical characteristics of medical devices, and tissue allografts, especially injectable biologics.
\end{abstract}

Key words: gamma radiation, sterilization, medical devices, tissue grafts, micronized amniotic membrane injections, food, detrimental effects

\section{Introduction}

Medical materials, tissue allografts and food samples must be sterilized prior to their use (1). For this purpose, dry heat, ethylene oxide (EtO), formaldehyde, gas plasma, peracetic acid, e-beams and gamma rays, are usually utilized (2-4). Which of these sterilization methods will be used depends on the purpose and physico-chemical properties of sterilized sample (2-4). Gamma radiation has several advantages over other sterilization methods: better penetration, better certainty of sterility, effectiveness independent of temperature and pressure conditions (5-6). Although gamma radiation is broadly used for the sterilization of medical equipment, micronized amniotic membrane injectable products and food samples (5-7), there are many proofs demonstrating deleterious effects of gamma radiation on sterilized products.

Herewith, we summarized current knowledge regarding molecular mechanisms responsible for detrimental effects of gamma radiation on sterilization with focusing on its significance for food safety and biomechanical characteristics of medical devices and tissue allografts.

\section{Molecular mechanisms involved in gamma rays-induced cell damage}

Morphological and functional changes, observed in irradiated products, are happening due to the adsorption of energy released during gamma 
radiation (7). Several hypotheses attempted to explain the mechanism of gamma rays-induced cell injury (8-12): increased permeability of cellular membrane (8), dysfunction of enzymes (9) and generation of radiotoxins (10). Regardless of the fact that these hypotheses are well documented, it is now widely accepted, based on the significant number of experimental proofs, that damage of deoxyribonucleic acid (DNA) is mainly responsible for detrimental effects of gamma radiation (11-12). Gamma rays either destruct DNA helix directly, or generate free radicals which disrupt chemical bonds within DNA (11-12).

\section{Resistance of microorganisms to gamma radiation}

Resistance of microorganisms to gamma radiation is mainly dependent on the microorganisms' capability to repair single strand breaks due to the activity of their DNA repair enzymes (13). Strains which don't have this competence are a lot more radiosensitive than the others (14). Viruses are more resistant to radiation than bacterial spores, and those are more resilient than vegetative bacteria, yeasts and molds. Parasites and helmints are more radio-resistant then bacteria: high doses of radiation (4-6kGy) have to be used for their elimination.

It is important to highlight that gamma rays may induce cell death of parasites or can interfere with their life cycle significantly affecting their pathogenicity (15). For example, by preventing completion of their life cycle, low dose of gamma radiation impairs transmissibility of parasitic protozoa and attenuates their infectivity $(7,15)$.

Interestingly, there is notable variability in radiation resistance within the same type of microorganisms. For example, yeasts are more immune to radiation than molds. Single stranded DNA viruses have higher radiation sensitivity compared to double stranded ones. The same was true for aerobic and non-pathogenic Bacillus spores when compared to anaerobic and toxigenic Clostridium spores. Higher radiation resistance of Gram-positive bacteria, in comparison to Gram-negative ones, indicate the importance of peptidoglycan for bacterial resistance to detrimental effects of gamma rays (15).

Since temperature and water content has significant role in the radio-sensitivity, higher doses of gamma rays have to be used for the elimination of bacteria and parasites from frozen food or dry biological products (15-16). Decrease in temperature remarkably reduce diffusion of free radicals in irradiated water and significantly attenuates their capacity to reach and damage DNA. Similarly, very small number of water-derived free radicals are formed when dry biological samples are irradiated, allowing higher radiation resistance to the microorganisms that are present in these samples $(7$, 15).

During incomplete sterilization, all microorganisms are not eliminated from irradiated product. In these cases, several residual microorganisms proceed to divide and, after two weeks, reach the identical population levels as in the non-irradiated samples. Accordingly, if patient consumes incompletely decontaminated food, the onset of symptoms is postponed making the diagnosis perplex. Importantly, progeny of microorganisms which outlived radiation are more resistant to gamma rays than the population from which they were originated (17). For instance, Deinococcus radiodurans which is the most familiar radiation-resistant organism, was isolated from cans of meat subjected to irradiation (18).

\section{Detrimental effects of sterilization by gamma radiation on tissue allografts}

Despite the fact that processing, preservation and storage of tissue allografts are always performed under strict aseptic conditions, possible presence of contaminating microorganisms indicates necessity of terminal/secondary sterilization by gamma irradiation (16). However, substantial and growing body of evidence demonstrated that sterilization by gamma rays resulted with alterations in morphological and functional characteristics of bone, tendon, tracheal, skin and amnion tissue grafts.

The potential of bone allografts to take part in the process of new bone remodeling is significantly reduced after sterilization by gamma rays. When bone allografts are irradiated, their structural components, like collagen fibers, break-down, which leads to the significant reduction of ultimate strength, toughness and resistance to crack propagation (19-28). These deleterious effects of gamma rays are dose dependent. The standard dose of $25 \mathrm{kGy}$ reduces bone strength for $20-30 \%$ (21), and every further increase in irradiation dose for $5 \mathrm{kGy}$ results in augmentation of strength's reduction for additional 25\% (22). Notably, work-to-fracture of irradiated bone grafts are decreased by $70 \%$ (21), supporting the fact that gamma rays remarkably reduce bone toughness of sterilized allografts. In line with these findings are results obtained by Mitchell and coworkers (23) showing that fatigue life was significantly reduced when bone grafts were sterilized with high dose of gamma radiation $(36.4 \mathrm{kGy})$. This information is particularly important having in mind that high dose of gamma radiation has to be used for the elimination of radio-resistant microorganisms (double stranded 
DNA viruses) (20-21). Opposite findings were reported in two trials which investigated therapeutic potential of bone allografts irradiated by high dose of gamma rays (24-25). While Lietman and co-workers noticed significantly higher fracture rate in patients that were implanted with irradiated grafts compared to those that received non-irradiated grafts (38\% vs. $18 \%)(24)$, Hernigou and colleagues reported fractures in only $6 \%$ of transplanted irradiated bone grafts (25).

The effects of low doses of irradiation (below $25 \mathrm{kGy}$ ) on biomechanical characteristic of bone grafts are not completely known since only few studies explored this issue and obtained results were inconsistent since several variables including age, gender and anatomical position of transplanted grafts were different in these trials (20). Therefore, new studies should be conducted under strictly controlled conditions in order to determine in what extent low doses of gamma rays affect morpho-functional properties of bone grafts.

In addition to reduced biomechanical properties, bone allografts sterilized by gamma rays had attenuated capacity for bone remodeling (26-28). Gamma rays induced degradation of growth factors which were crucially involved in osteoinduction (bone morphogenetic protein and transforming growth factor- $\beta$ ) (26). Moreover, gamma radiation altered function of irradiated osteoclasts and osteoblasts. Irradiated osteoclasts had reduced capacity for bone resorption (27) while irradiated osteoblasts were not able to optimally produce bone matrix (28).

Similar as bone allografts, tendon allografts were also susceptible to the gamma rays-induced changes in mechanical properties after sterilization with standard or high dose of gamma radiation (29). Stiffness and failure loads of tendon allografts were mainly affected by irradiation. These findings were noticed in clinical study that investigated therapeutic potential of irradiated hamstring tendon allografts for the reconstruction of anatomic double-bundle anterior cruciate ligament. A significant increase in anterior and rotational laxity was observed in patients that received irradiated hamstring grafts when compared to the patients that were implanted with non-irradiated allografts (30), confirming deleterious effects of gamma rays on mechanical characteristics of tendon allografts.

Transplantation of stem cell-repopulated decellularized tracheal grafts is nowadays the most effective therapeutic procedure for patients with tracheal loss (31). It was hypothesized that gamma radiation may be used for sterilization of decellularized tracheal grafts. However, standard dose of gamma rays induced degradation of collagen fibers and significantly changed architecture of decellularized tracheal grafts in manner that stem cell repopulation was difficult to conduct (31).

Sterilization by gamma radiation also reduced therapeutic potential of skin and amnion tissue grafts (32). Structural changes in basement membrane, elastic and collagen fibers were observed in irradiated skin grafts (32). Low dose of gamma radiation (12.5 kGy) induced vacuolar cytoplasmic changes in epidermal cells while fine elastic fibers were more radio-resistant and their damage was observed only after sterilization with standard dose of irradiation (25 kGy). Similar harmful effects of sterilization by gamma rays were confirmed by chromatin condensation which was observed in irradiated amnion grafts (32).

\section{Consumption of irradiated food: what have we learnt from animal models?}

Infestation of food with pathogenic microorganisms, is large-scaled public health problem and cause of enormous global economic losses. Exclusion of food borne pathogens is of great importance for people with impaired immune response: elders, patients with primary or secondary immunodeficiency and those who undergo chemo or radiotherapy (7). Thermal pasteurization is successfully used for elimination of microorganisms from liquid foods, but is not as efficient with sold and dry ingredients. Accordingly, gamma rays are utilized for this purpose. Since radiation can inactivate microorganisms in frozen foods without defrosting, it can be used for sterilization of final packed product ensuring that the irradiated product remains sterile until the package removal (7). However, results obtained in several experimental studies indicate that consumption of irradiated food may be detrimental for human health.

Consumption of irradiated food inhibited growth of young rats and analysis of their livers, kidneys, stomachs and guts revealed notable amounts of radioactivity (33-34). Gamma radiation induces generation of free radicals which modify structure and/or activity of vitamins significantly reducing nutritive value of irradiated food $(17,35)$. Moreover, because of the altered structure, vitamins from irradiated food are not well absorbed which leads to their deficiency (36). For instance, increased mortality was noticed among mice that consumed irradiated beef as a consequence of internal hemorrhage which developed due to the deficiency of vitamin $K$ (36). Similarly, second litter of the rats that were fed with irradiated beef had symptoms of nutritional muscular dystrophy (facial swelling, ruffled hair coat, loss of coordination and movement) due to the deficiency of 
vitamin E (37).

Irradiation of food that contains unsaturated fats generates peroxide which oxidize and polymerize lipids into non-digestible polymers which, as insoluble plaques, make depositions in the blood vessels (17). Accordingly, mice, rats and dogs that were, for 2 years or four generations, fed with irradiated food containing unsaturated fats developed atherosclerosis and high blood pressure and some of them died due to the heart rupture and massive hemorrhaging (17).

When food which contains unsaturated fats or nitrates is irradiated, well-known human cancerogens (benzopyrene quinone and nitrosamines) are generated as a consequence of gamma rays-induced chemical transformations of lipids or nitrates (38).

Results obtained in preclinical and clinical studies strongly suggest that consumption of irradiated food may induce chromosomal abnormalities and lethal mutations in rapidly dividing cells (39-48). An addition of irradiated sucrose to the growth medium resulted with genome instability in human lymphocytes (39). Chromosomes of these lymphocytes were grossly damaged (appeared broken or fragmented) and their mitosis were suppressed (39). Similarly, fragmentation of chromosomes accompanied with inhibited mitosis were noticed in human leucocytes when standard growth medium was replaced by irradiated one (40).

Similar results were observed in animal studies (41-47). An increased number of embryonic deaths, due to lethal mutations and gross chromosomal aberrations, was noticed among litters of mice that consumed irradiated food (41-42). Higher number of polyploidy cells in bone marrow and significant amount of aneuploidy cells in testis accompanied by increased mutagenic index was noticed in mice, rats and hamsters that were fed with irradiated wheat compared to the animals that were on standard diet (44-47). In line with these preclinical findings were results obtained in clinical trial demonstrating that consumption of irradiated wheat induced gross chromosomal polyploidy in malnourished Indian children (43).

Consumption of irradiated food affected immune response, as well. Lymphopenia, reduced number of plasmocytes in the splenic follicles and lower mean antibody titres were observed in rats that were fed with irradiated wheat (49).

Having in mind that chromosome number return to normal several weeks after discontinuation of irradiated diet (44-49), it seems that detrimental effects of radiation on chromosome structure and integrity were temporary and reversible. However, since genetic instability in polyploidy cells may represent an initial step of oncogenesis, findings that consumption of irradiated food may result with polyploidy have to be carefully explored in future long-term prospective clinical studies with significant number of participants. This is of particular importance since all previously described animal studies $(41-47,49)$ were, due to the life span of experimental animals, too short to indicate the possibility of the increased incidence of cancer while the only clinical trial that investigated this issue (48) recruited small number of participants. Accordingly, at this moment, based on these findings, we can just assume that consummation of irradiated food could contribute to the development of cancer by provoking genome instability. For confirmation of this hypothesis, since oncogenesis is long-lasting process, we should wait at least several decades to demonstrate an increased cancer incidence among people who consumed irradiated food.

\section{Structural changes seen in polymer medical devices sterilized by gamma rays}

During sterilization, structural characteristics of polymer medical devices are considerably modified by gamma radiation (50-57). Since these irradiation-induced effects are cumulative, structural changes were usually observed in devices which were sterilized several times (50).

Polymethyl methacrylate (PMMA), ultrahigh molecular weight polyethylene (UHMWPE) polyvinyl chloride (PVC) and silicone rubber are radio-sensitive polymers and sterilization by standard dose of gamma radiation (25 $\mathrm{kGy})$ induces irreversible structural changes in these materials. Gamma radiation induces abstraction of hydrogen from an a-methyl or methylene groups in PMMA (51) and generates free radicals in UHMPWE (52) leading to the development of irreversible structural changes in PMMA and UHMWPE containing devices affecting their clinical use (51). Also, due to the activity of free radicals, silicone rubber-containing medical devices that were sterilized by gamma rays have reduced elasticity and limited use (53). Similarly, crosslinking and main chain scissions were noticed in 25 kGy-irradiated PVC containing medical devices affecting their clinical potential (54).

Physical characteristics of polycarbonate (PC) and polypropylene (PP) are significantly altered by gamma rays which significantly limits clinical use of irradiated PC and PP containing medical devices (55-56). PC is material which offers glasslike clarity to medical instruments enabling their utilization in diagnostic and therapeutic procedures in which visibility of tissues is crucially important (55). Since $\mathrm{PC}$ is not radio-sensitive material, only high doses of 
gamma rays generates phenoxy and phenyl polymeric radicals which cause yellowness of PC containing devices significantly reducing their clarity and clinical use (55). Similarly, morphology, viscosity and melting temperature of PP are remarkably altered after sterilization by gamma radiation (56). These changes are particularly noticeable when PP is sterilized by gamma rays in the air, as a consequence of the oxidative destruction of PP structure (56).

In addition to the alteration of its physico-chemical characteristics, gamma rays can induce generation of a carcenogen 4,4'-methylenedianiline in polyurethane (PU). Accordingly, gamma rays should not be used for the sterilization of PU containing medical devices due to the safety issues (57).

\section{Conclusions}

The standard sterilization method for many medical devices and food samples over the past 40 years involves gamma radiation. However, when applying gamma radiation for sterilization of tissue allografts and polymer medical devices, structural changes are induced. These changes have the potential to adversely affect the clinical use of irradiated products. Accordingly, additives that will interrupt degradable reactions initiated by gamma rays, should be used during sterilization for the protection from detrimental effects of gamma radiation. Moreover, gamma radiation has been shown to generate free hydroxyl radicals and other radiotoxins. This adds additional risks of both toxigenic and mutagenic effects which could potentially induce cancer. Since several animal studies demonstrated that consummation of irradiated food provoked genome instability, new, long-term, prospective clinical studies should be conducted in near future to investigate whether irradiated food is safe for human consumption.

In conclusion, more safety studies are needed to clearly define the risks of gamma radiation method of sterilization. The increased interest in newer biologics emphasizes the need for more modern sterilization methods avoiding all potential risks that gamma radiation can cause to health.

\section{Acknowledgments}

This work was supported by Swiss National Science Foundation project (SCOPES IZ73Z0_ 152454/1), Novartis foundation for medical-biological research (Grant No.16C197), Faculty of Medical Sciences University of Kragujevac (JP 02/09) and Serbian Ministry of Science (ON175069 and ON175103).

\section{Competing Interests}

The authors have declared that no competing interest exists.

\section{References}

1. Rutala WA, Weber DJ. Disinfection and Sterilization in Health Care Facilities: An Overview and Current Issues. Infect Dis Clin North Am. 2016; 30:609-637.

2. Shintani H. Ethylene Oxide Gas Sterilization of Medical Devices. Biocontrol Sci. 2017; 22:1-16.

3. Lambert BJ, Mendelson TA, Craven MD. Radiation and ethylene oxide terminal sterilization experiences with drug eluting stent products. AAPS PharmSciTech. 2011; 12:1116-1126.

4. Alvarado CJ. Sterilization vs. disinfection vs. clean. Nurs Clin North Am. 1999; 34:483-491.

5. Singh R, Singh D, Singh A. Radiation sterilization of tissue allografts: A review. World J Radiol. 2016; 8:355-369.

6. Brinston RM, Wilson BK. Converting to gamma-radiation sterilization: an overview for medical device manufacturers. Med Device Technol. 1993; 4:18-22.

7. Farkas J. Irradiation as a method for decontaminating food. A review. Int J Food Microbiol. 1998; 44:189-204.

8. AlZahrani K, Al-Sewaidan HA. Nanostructural Changes in the Cell Membrane of Gamma-Irradiated Red Blood Cells. Indian J Hematol Blood Transfus. 2017; 33:109-115.

9. Rendic S, Guengerich FP. Summary of information on the effects of ionizing and non-ionizing radiation on cytochrome P450 and other drug metabolizing enzymes and transporters. Curr Drug Metab. 2012; 13:787-814.

10. Ibragimova MI, Petukhov VY, Zheglov EP, Khan N, Hou H, Swartz HM, Konjukhov GV, Nizamov RN. Quinoid radio-toxin (QRT) induced metabolic changes in mice: an ex vivo and in vivo EPR investigation. Nitric Oxide. 2008; 18:216-222.

11. Sage E, Shikazono N. Radiation-induced clustered DNA lesions: Repair and mutagenesis. Free Radic Biol Med. 2017; 107:125-135.

12. Kuefner MA, Brand M, Engert C, Schwab SA, Uder M. Radiation Induced DNA Double-Strand Breaks in Radiology. Rofo. 2015; 187:872-878.

13. Broomfield S, Hryciw T, Xiao W. DNA postreplication repair and mutagenesis in Saccharomyces cerevisiae. Mutat Res. 2001; 486:167-184.

14. Pavlopoulou A, Savva GD, Louka M, Bagos PG, Vorgias CE, Michalopoulos I, Georgakilas AG. Unraveling the mechanisms of extreme radioresistance in prokaryotes: Lessons from nature. Mutat Res Rev Mutat Res. 2016; 767:92-107.

15. Halls N. The microbiology of irradiation sterilization. Med Device Technol. 1992; 3:37-45.

16. Grieb TA, Forng RY, Stafford RE, Lin I, Almeida I, Bogdansky S, Ronholdt C, Drohan WN, Burgess WH. Effective use of optimized, high-dose (50 kGy) gamma irradiation for pathogen inactivation of human bone allografts. Biomaterials. 2005; 26:2033-2042.

17. Tritsch GL. Food irradiation. Nutrition. 2000; 16:698-701.

18. White O, Eisen JA, Heidelberg JF, Hickey EK, Peterson JD, Dodson RJ, Haft DH, Gwinn ML, Nelson WC, Richardson DL, Moffat KS, Qin H, Jiang L, Pamphile W, Crosby $M$, Shen M, Vamathevan JJ, Lam P, McDonald L, Utterback T, Zalewski C, Makarova KS, Aravind L, Daly MJ, Minton KW, Fleischmann RD, Ketchum KA, Nelson KE, Salzberg S, Smith HO, Venter JC, Fraser CM.. Genome sequence of the radioresistant bacterium Deinococcus radiodurans R1. Science 1999; 286:1571.

19. Triantafyllou N, Sotiropoulos E, Triantafyllou JN. The mechanical properties of the lyophylized and irradiated bone grafts. Acta Orthop Belg. 1975; 41:35-44.

20. Nguyen H, Morgan DA, Forwood MR. Sterilization of allograft bone: effects of gamma irradiation on allograft biology and biomechanics. Cell Tissue Bank. 2007; 8:93-105.

21. Currey JD, Foreman J, Laketić I, Mitchell J, Pegg DE, Reilly GC. Effects of ionizing radiation on the mechanical properties of human bone. J Orthop Res. 1997; 15:111-117.

22. Godette GA, Kopta JA, Egle DM. Biomechanical effects of gamma irradiation on fresh frozen allografts in vivo. Orthopedics. 1996; 19:649-653.

23. Mitchell EJ, Stawarz AM, Kayacan R, Rimnac CM. The effect of gamma radiation sterilization on the fatigue crack propagation resistance of human cortical bone. Bone Joint Sur.2004; 86:2648-2657.

24. Lietman AS, Tomford WW, Gebhardt CM, Springfield SD, Mankin JM. Complications os irradiated allografts in orthopaedic tumor surgery. J Clin Orthopaedic Relat Res. 2000; 375:214-217.

25. Hernigou P, Delepine G, Goutallier D, Julieron A. Massive allografts sterilised by irradiation. Clinical results J Bone Joint Surg Br. 1993; 75:904-913.

26 Ijiri S, Yamamuro T, Nakamura T, Kotani S, Notoya K. Effect of sterilization on bone morphogenetic protein. J Orthop Res. 1994; 12:628-636.

27. Kluger R, Bouhon W, Freudenberger H, Kröner A, Engel A, Hoffmann O. Removal of the surface layers of human cortical bone allografts restores in vitro osteoclast function reduced by processing and frozen storage. Bone. 2003; 32:291-296. 
28. Schuster JM, Avellino AM, Mann FA, Girouard AA, Grady MS, Newell DW, Winn HR, Chapman JR, Mirza SK. Use of structural allografts in spinal osteomyelitis: a review of 47 cases. J Neurosurg. 2000; 93:8-14.

29. Hoburg A, Keshlaf S, Schmidt T, Smith M, Gohs U, Perka C, Pruss A, Scheffler S. High-dose electron beam sterilization of soft-tissue grafts maintains significantly improved biomechanical properties compared to standard gamma treatment. Cell Tissue Bank. 2015; 16:219-226.

30. Tian S, Ha C, Wang B, Wang Y, Liu L, Li Q, Yang X, Sun K. Arthroscopic anatomic double-bundle ACL reconstruction using irradiated versus non-irradiated hamstring tendon allograft. Knee Surg Sports Traumatol Arthrosc. 2017; 25:251-259

31. Johnson CM, Guo D, Ryals S, Postma GN, Weinberger PM. The feasibility of gamma radiation sterilization for decellularized tracheal grafts. Laryngoscope. 2017; 127:E258-E264.

32. Mrázová $H$, Koller J, Kubišová K, Fujeríková G, Klincová E, Babál P. Comparison of structural changes in skin and amnion tissue grafts for transplantation induced by gamma and electron beam irradiation for sterilization. Cell Tissue Bank. 2016; 17:255-60.

33. Tinsley IJ, Bone JF, Bubl EC. The growth, reproduction, longevity, and histopathology of rats fed gamma-irradiated carrots. Toxicol Appl Pharmacol. 1970; 16:306-317.

34. De AK, Aiyar AS, Sreenivasan A. Biochemical effects of irradiated sucrose solutions in the rat. Radiat Res. 1969; 37:202-215.

35. Mellette SJ, Leone LA. Influence of age, sex, strain of rat and fat soluble vitamins on hemorrhagic syndromes in rats fed irradiated beef. Fed Proc. 1960; 19:1045-1049.

36. Metta VA, Mameesh MS, Johnson BC. Vitamin K deficiency in rats induced by the feeding of irradiated beef. J Nutr. 1959; 69:18-22.

37. Poling C, Warner W, Humberg F, Reber E, Urbain W, Rice E. Growth, reproduction, survival and histopathology of rats fed beef irradiated with electrons. Food Res. 1955; 20:193-214.

38. Wakabayashi K, Nagao M, Chung TH, Yin MQ, Karai I, Ochiai M, Tahira T, Sugimura T. Appearance of direct-acting mutagenicity of various foodstuffs produced in Japan and Southeast Asia on nitrite treatment. Mutat Res. 1985; 158:119-124.

39. Shaw MW, Hayes E. Effects of irradiated sucrose on the chromosomes of human lymphocytes in vitro. Nature. 1966; 211:1254-1255

40. Kesavan, PC, Swaminathan MS. Cytotoxic and radiomimetic activity of irradiated culture medium on human leukocytes. Current Science. 1966; 16:403-404.

41. Moutschen-Dahmen M, Moutschen J, Ehrenberg L. Pre-implantation death of mouse eggs caused by irradiated food. Int J Radiat Biol Relat Stud Phys Chem Med. 1970; 18:201-216.

42. Anderson D, Clapp MJ, Hodge MC, Weight TM. Irradiated laboratory animal diets: dominant lethal studies in the mouse. Mutat Res. 1981; 80:333-345.

43. Bhaskaram C, Sadasivan G. Effects of feeding irradiated wheat to malnourished children. Am J Clin Nutr 1975; 28:130.

44. Vijayalaxmi. Genetic effects of feeding irradiated wheat to mice. Can J Genet Cytol. 1976; 18:231-238.

45. Vijayalaxmi, Sadasivan G. Chromosomal aberrations in rats fed irradiated wheat. Inter Journ Rad Biol, 1975; 27:135-142

46. Vijayalaxmi, Rao KV. Dominant lethal mutations in rats fed on irradiated wheat. Inter Journ Rad Biol, 1976; 29:93-98.

47. Renner HW. Chromosome studies on bone marrow cells of chinese hamsters fed a radiosterilized diet. Toxicology. 1977; 8:213-222.

48. Vijayalaxmi. Cytogenetic studies in monkeys fed irradiated wheat. Toxicology. 1978; 9:181.

49. Vijayalaxmi. Immune response in rats given irradiated wheat. British Journal of Nutrition. 1978; 40:535-541.

50. Da Silva F, Aquino K, Araujo E. Effects of gamma irradiation on poly(vinyl chloride)/polystyrene blends: Investigation of radiolytic stabilization and miscibility of the mixture. Polymer Degradation and Stability. 2008; 93: 2199-2203.

51. Cehreli MC, Sahin S, Ergunay K, Ustacelebi S, Sevil UA. Cytotoxicity of eluates from a gamma-ray-polymerized poly(methyl methacrylate). J Biomater Appl. 2004; 18:223-231.

52. Goldman M, Gronsky R, Pruitt L. The influence of sterilization technique and ageing on the structure and morphology of medical-grade ultrahigh molecular weight polyethylene. Journal of Materials Science: Materials in Medicine. 1998; 9: 207- 212.

53. Traeger R, Castonguay T. Effect of $\gamma$-radiation on the dynamic mechanical properties of silicone rubbers. Journal of Applied Polymer Science. 1996; 10:535-550.

54. Vinhas G, Souto-Maior RM, de Almeida YMB, Neto BB. Radiolytic degradation of poly(vinyl chloride) systems. Polymer Degradation and Stability. 2004; 86: 431-436.

55. Araujo E, Khoury H, Silveira S. Effects of gamma-irradiation on some properties of durolon polycarbonate. Radiation Physics and Chemistry. 1998; 53: 79-84.

56. Khang G, Lee HB, Park JB. Radiation effects on polypropylene for sterilization. Biomed Mater Eng. 1996; 6:323-334.

57. Shintani H. Formation and elution of toxic compounds from sterilized medical products: methylenedianiline formation in polyurethane. J Biomater Appl. 1995; 10:23-58. 